\title{
Leaf shape in Populus tremula is a complex, omnigenic trait.
}

Niklas Mähler ${ }^{1}$, Bastian Schiffthaler ${ }^{1}$, Kathryn Robinson ${ }^{1}$, Barbara Terebieniec ${ }^{1}$, Matej Vucak $^{2}$, Mark Bailey ${ }^{2}$, Chanaka Mannapperume ${ }^{1}$, Stefan Jansson ${ }^{1}$, Torgeir R Hvidsten ${ }^{3}$, and Nathaniel Street ${ }^{1,1}$

${ }^{1}$ Umeå University

${ }^{2}$ University of Glasgow

${ }^{3}$ Norwegian University of Life Sciences

May 5, 2020

\begin{abstract}
Leaf shape is a defining feature of how we recognise and classify plant species. Although there is extensive variation in leaf shape within many species, few studies have disentangled the underlying genetic architecture. We characterised the genetic architecture of leaf shape variation in Eurasian aspen (Populus tremula L.) by performing a genome wide association studies (GWAS) for physiognomy traits. To ascertain the roles of identified GWAS candidate genes within the leaf development transcriptional program, we performed gene co-expression network analyses from a developmental series, which is publicly available at http://aspleaf.plantgenie.org. We additionally used gene expression measurements across the population to analyse GWAS candidate genes in the context of a population-wide co-expression network and to identify genes that were differentially expressed between groups of individuals with contrasting leaf shapes. These data were integrated with expression GWAS (eQTL) results to define a set of candidate genes associated with leaf shape variation. Our results identified no clear adaptive link to leaf shape variation and indicate that leaf shape traits are genetically complex, likely determined by numerous small-effect variations in gene expression. Genes associated with shape variation were peripheral within the population-wide co-expression network, were not highly connected within the leaf development co-expression network and exhibited signatures of relaxed selection. As such, our results are consistent with the omnigenic model.
\end{abstract}

\section{Leaf shape in Populus tremula is a complex, omnigenic trait.}

Running title: Omnigenic genetic architecture of aspen leaf shape

Niklas Mähler $^{1}$, Bastian Schiffthaler ${ }^{1}$, Kathryn M. Robinson ${ }^{1+}$, Barbara K. Terebieniec ${ }^{1+}$, Matej Vučak ${ }^{2}$, Chanaka Mannapperuma ${ }^{1}$, Mark E.S. Bailey ${ }^{2}$, Stefan Jansson ${ }^{1}$, Torgeir R. Hvidsten ${ }^{3}$, Nathaniel R. Street ${ }^{* 1}$

+ Equal contribution

* Corresponding author: Nathaniel R. Street: nathaniel.street@umu.se

${ }^{1}$ Umeå Plant Science Centre, Department of Plant Physiology, Umeå University, 90187 Umeå, Sweden.

${ }^{2}$ School of Life Sciences, College of Medical, Veterinary and Life Sciences, University of Glasgow, G12 8QQ, Glasgow, Scotland.

${ }^{3}$ Faculty of Chemistry, Biotechnology and Food Science, Norwegian University of Life Sciences, 1432 As, Norway.

Niklas Mähler: niklas.mahler@umu.se 
Bastian Schiffthaler: bastian.schiffthaler@umu.se

Barbara K. Terebieniec: terebieniec.barbara@googlemail.com

Kathryn M. Robinson: kathryn.robinson@umu.se

Matej Vučak: matej.vucak@glasgow.ac.uk

Mark E.S. Bailey: mark.bailey@glasgow.ac.uk

Chanaka Mannapperume: chanaka.mannapperume@umu.se

Stefan Jansson: stefan.jansson@umu.se

Torgeir R. Hvidsten: torgeir.r.hvidsten@nmbu.no

\begin{abstract}
Leaf shape is a defining feature of how we recognise and classify plant species. Although there is extensive variation in leaf shape within many species, few studies have disentangled the underlying genetic architecture.

We characterised the genetic architecture of leaf shape variation in Eurasian aspen (Populus tremula L.) by performing a genome wide association studies (GWAS) for physiognomy traits. To ascertain the roles of identified GWAS candidate genes within the leaf development transcriptional program, we performed gene co-expression network analyses from a developmental series, which is publicly available at http://aspleaf.plantgenie.org. We additionally used gene expression measurements across the population to analyse GWAS candidate genes in the context of a population-wide co-expression network and to identify genes that were differentially expressed between groups of individuals with contrasting leaf shapes. These data were integrated with expression GWAS (eQTL) results to define a set of candidate genes associated with leaf shape variation.

Our results identified no clear adaptive link to leaf shape variation and indicate that leaf shape traits are genetically complex, likely determined by numerous small-effect variations in gene expression. Genes associated with shape variation were peripheral within the population-wide co-expression network, were not highly connected within the leaf development co-expression network and exhibited signatures of relaxed selection. As such, our results are consistent with the omnigenic model.
\end{abstract}

Keywords: Leaf shape; Populus tremula; natural variation, GWAS, complex trait, omnigenic

\title{
Introduction
}

Leaf shape is a defining feature of how we recognise and classify plant species and, as such, is an important component of our relationship with nature. Floral morphology and features are largely invariant within species and therefore served as the basis for taxonomic classification. In contrast, leaf shape varies distinctly between and, often extensively, within species. There are some identifiable global trends, indicating convergent evolution, such as a narrowing and more defined serration of leaves toward latitudinal extremes (Peppe et al., 2011; Royer, McElwain, Adams, \& Wilf, 2008; Royer, Wilf, Janesko, Kowalski, \& Dilcher, 2005; Traiser, Klotz, Uhl, \& Mosbrugger, 2005). Despite the vast diversity of leaf shapes represented across plant species, our knowledge of the genetic architecture of natural variation in leaf shape, the evolutionary drivers and adaptive significance of that variation or the molecular control of leaf development remains relatively limited (D. H. H. Chitwood \& Sinha, 2016; Ichihashi et al., 2014).

Leaf organogenesis is initiated within an apical meristem from a group of dividing, undifferentiated initial (meristematic) cells (Clark, 1997). As the meristematic cells divide, the daughter cells reach the meristem peripheral zone and enter determinate growth. Once this initiation phase is complete, a boundary region between the meristem and the outgrowing lateral organ is established (Sluis \& Hake, 2015), followed by 
expansion of leaf primordia (Cleland, 2001). Leaf growth is a tightly coordinated process involving both cell proliferation (division) and expansion (Czesnick \& Lenhard, 2015), both of which are coordinated by polar hormone distributions (Moon \& Hake, 2011; Sluis \& Hake, 2015). Leaf morphogenesis continues with growth in the proximo-distal, adaxial-abaxial, and medial-lateral axes, with polarised cell division and expansion along each axis creating a flat leaf lamina and with spatially varying rates of expansion and division determining basic leaf shape (Moon \& Hake, 2011; Tsukaya, 2005).

A number of genes with a central role in the control of leaf primordia initiation and subsequent leaf development and pattern formation have been identified from forward genetic screens, largely inArabidopsis thaliana (Tsukaya, 2005). For example, genes including the NAC transcription factors NO APICAL MERISTEM and CUP SHAPED COTYLEDONS (CUC), are expressed at the boundary region to delineate outgrowing leaf primordia from the meristem (Aida, Ishida, Fukaki, Fujisawa, \& Tasaka, 1997; Cheng et al., 2012; Souer, van Houwelingen, Kloos, Mol, \& Koes, 1996; Vroemen, Mordhorst, Albrecht, Kwaaitaal, \& de Vries, 2003). Most classically, ANGUSTIFOLIA (AN) and ROTUNDIFOLIA (ROT) act independently to control polar length and width expansion, respectively (Tsuge, Tsukaya, \& Uchimiya, 1996). There are also a number of notable examples of large-effect genes underlying variation in leaf complexity (dissection), such as the A. thaliana HD-zip transcription factorLATE MERISTEM IDENTITY1 (LMI1) homolog underlying leaf morphs in cotton (Andres et al., 2017).

Although forward genetic screens have identified genes acting during leaf organogenesis, most studies have been conducted using $A$. thaliana mutants that target single genes. Although those genes can be shown to be essential for, or to contribute to, the control of leaf development, they are not necessarily those underlying natural variation. Leaf shape is a complex, multigenic trait (D. H. Chitwood, Kumar, et al., 2013; D. H. Chitwood, Ranjan, Martinez, et al., 2014; Gupta, Rosenthal, Stinchcombe, \& Baucom, 2019), meaning that causal variation in protein coding sequence or expression of each contributing gene will be of small effect size in relation to the total variation in the population. As such, alternative approaches are needed to identify loci underlying natural variation. One such approach is to use genetic screens to identify genomic regions involved in the control of complex traits. For example, Genome Wide Association Studies (GWAS) or Quantitative Trait Locus (QTL) mapping can be used, with the results being integrated with targeted expression studies or used to select candidates for forward genetics validation. A number of studies have used such integrative approaches to study leaf shape. Xiao et al.(2014) used a systems genetics approach to study the genetic architecture of leaf development in a Brassica rapa double haploid population, identifying a cohort of candidate genes. Those candidates included well-characterised examples such as AINTIGUMENTA (ANT ), CUC2 and GIBBERELIN 20-OXIDASE 3 (GA20OX3) in addition to numerous genes with no currently assigned function during leaf development. Tight genetic regulation of leaf traits has also been reported in Vitis vinifera, with grape leaves displaying large-scale morphological variation among cultivars. Chitwood et al. (2014) and Gupta et al. (2019) showed that many leaf shape traits, including venation patterning, are highly heritable and that genes displaying differential expression can be identified between cultivars with contrasting leaf shapes. A number of studies of leaf shape have similarly been performed using tomato introgression lines and backcross inbred line populations of crosses between domesticated tomato (Solanum lycopersicum ) and wild Solanum spp. (D. H. Chitwood, Kumar, et al., 2013; D. H. Chitwood, Ranjan, Kumar, et al., 2014; Fulop et al., 2016), including transcriptional studies (D. H. Chitwood, Maloof, \& Sinha, 2013; Ichihashi et al., 2014; Ranjan et al., 2016). Phenotypic and expression GWAS in A. thaliana and transcription profiling in recombinant inbred lines of Zea mays have shown that the balance of cell division and expansion determining leaf growth differs among genetic backgrounds (Clauw et al., 2016).

Populus is an important model system for genomics, ecological and evo-devo studies. Populus species were appropriately described by Stettler \& Bradshaw (1996) as being "replete with variation", a statement that is particularly relevant to their extensive variation in leaf shape. Within the genus, the aspen species $P$. tremula and $P$. tremuloides, for example, contain extensive intra-specific natural variation in leaf shape (Barnes, 1969, 1975; Bylesjö et al., 2008), heterophylly along extending long shoots (Cox, 2005; Curtis \& Lersten, 1978) and striking heteroblasty between leaves produced at the extending shoot apical meristem and those of short shoot buds (Critchfield, 1960). The pre-formed leaves of most aspen species have flattened 
petioles that cause the characteristic trembling or quaking of the leaf lamina in very light wind. A number of QTL studies have been performed using hybrid Populus crosses, which have highlighted the polygenic control and heritability of leaf physiognomy traits (Wuet al. , 1997; Rae et al. , 2006; Lindtke et al. , 2013; Drost et al. , 2015). Using a pseudo-backcross pedigree of narrow-leaf $P$. trichocarpa (section Tacamahaca) and broad-leaved P. deltoides (section Aigeiros ) Drostet al. (2015) identified a major QTL for leaf lamina width and length:width ratio. The mapped locus contains an ADP-ribosylation factor (ARF), which is a strong candidate gene for the regulation of leaf morphology. Street et al. (2008, 2011) performed systems biology analyses to link available microarray expression data to leaf physiognomy QTLs, identifying GRF s (Growth-Regulating Factors) as candidate genes controlling leaf development. There are also a small number of reports of aberrant or altered leaf phenotypes resulting from genetic transformation studies (Du, Mansfield, \& Groover, 2009; Ratke et al., 2018; Rottmann et al., 2000).

We used a GWAS of physiognomy traits in aspen to demonstrate the complex genetic architecture underlying the observed phenotypic variation. Gene expression data were used to identify genes differentially expressed between groups of genotypes with contrasting leaf shapes and these were examined for the presence of SNPs associated with expression variation. To examine whether genes underlying natural variation in these complex traits were central within the developmental transcriptional program, we generated co-expression networks from developmental series of terminal and pre-formed leaves. We examined the developmental profiles of GWAS candidate genes and explored their co-expression network centrality within a leaf development co-expression network. Furthermore, we used a population gene co-expression network to characterise the centrality of candidate genes associated with shape variation in this network and to investigate signatures of selection. Taken together, the results from our analysis indicate that leaf shape variation in aspen is highly polygenic, and is associated with numerous small effect-size variants affecting genes expressed during leaf development.

\section{Materials and Methods}

\section{Leaf shape phenotyping in the Swedish Aspen collection}

Leaf size and shape parameters were measured in a natural population of Populus tremula, the Swedish Aspen (SwAsp) collection, growing in common gardens at Sävar, northern Sweden $\left(63.9^{\circ} \mathrm{N}, 20.5^{\circ} \mathrm{E}\right)$ and Ekebo, southern Sweden $\left(55.9^{\circ} \mathrm{N}, 13.1^{\circ} \mathrm{E}\right)$. The common garden trials comprised of natural (wild-growing) aspen genotypes collected in 2003 across ten latitudinal degrees, which were cloned and planted in 2004 in a randomised block design in each garden (Luquez et al., 2008). Leaf samples were harvested in Sävar on 14 July 2008 and 28 June 2011 and in Ekebo on 18 July 2008 and 4 August 2011, when leaves were fully expanded and mature, but before the occurrence of substantial damage due to herbivory or the presence of fungal rust infection. Ten undamaged leaves per replicate tree were sampled randomly across the canopy, avoiding leaves from the first or last leaf in a leaf cohort originating from a single bud. In total, in 430, 444, 326 and 393 trees were sampled in Ekebo 2008, Ekebo 2011, Sävar 2008 and Sävar 2011 respectively, comprising between 1 and $8($ median $=3)$ clonal replicates. One hundred and thirteen genotypes were sampled in both years in Ekebo and in 2011 in Sävar, and 111 genotypes were sampled in 2008 in Sävar. Leaves were stored at $4^{\circ}-8^{\circ} \mathrm{C}$ immediately after harvest. Petioles were removed at the leaf base and the sample of ten leaves per tree was scanned in colour at $300 \mathrm{dpi}$ using a CanoScan 4400F. A 5 x $4 \mathrm{~cm}$ Post-it note was scanned as a scale image. The resulting images were analysed using LAMINA (Bylesjö et al., 2008) to obtain leaf size and shape metrics. Median values of the ten leaves per tree were calculated for each leaf size and shape metric and the median value per individual was used for subsequent analyses, such as heritability and $\mathrm{QST}_{\mathrm{ST}}$.

\section{Statistical analyses}

All statistical analyses of the SwAsp collection physiognomy data were conducted in R. Phenotypic data were examined for homogeneity of variance. No data transformations were required to meet the assumptions of a normal distribution. Pearson correlations were used for all phenotypic correlations calculated. 
We calculated clonal repeatability $(\mathrm{R})$ and used this to provide an upper-bound estimate of broad sense heritability $\left(\mathrm{H}^{2}\right)$. We refer to this trait as $H^{2}$ rather than $\mathrm{R}$ as this probably allows a more intuitive interpretation for readers, however we note that the two are not the same (see Dohm, 2002 for discussion). Estimates of broad-sense heritability $\left(\mathrm{H}^{2}\right)$ and their $95 \%$ confidence intervals, including all clonal replicates, were calculated as

$\mathrm{H}^{2}=\mathrm{V}_{\mathrm{G}} /\left(\mathrm{V}_{\mathrm{G}}+\mathrm{V}_{\mathrm{E}}\right)$

where $V_{G}$ and $V_{E}$ are genetic and environmental variance components, using the heritability function in the $\mathrm{R}$ package 'Heritability'. To estimate population differentiation, $Q \mathrm{ST}$, the following formula was used:

$\mathrm{Q}_{\mathrm{ST}}=\mathrm{V}_{\mathrm{pop}} /\left(\mathrm{V}_{\text {pop }}+\left(2 * \mathrm{~V}_{\text {geno }}\right)\right)$

where $\mathrm{V}_{\text {pop }}$ and $\mathrm{V}_{\text {geno }}$ are the inter-population and genotype (i.e. inter-individual) genetic variance components, respectively.

Genetic correlations between phenotypes were calculated as

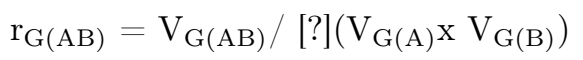

where $r_{G(A B)}$, the genetic correlation of phenotype $A$ and phenotype $B$, was calculated from the $V_{G(A B)}$, the genetic covariance in phenotype $A$ and phenotype $B, V_{G(A)}$ and $V_{G(B)}$ were the genetic variances of phenotypes $\mathrm{A}$ and $\mathrm{B}$ respectively.

Genetic (clonal) variation for each phenotype between years and common gardens was investigated using analyses of variance (ANOVA) where phenotype was the dependent variable and environment, comprising year and garden, the independent variables using the following model:

Phenotype Environment + Genotype + Environment x Genotype

ANOVA models were implemented in the aov function in $\mathrm{R}$. All effects were considered significant at $\mathrm{P}<0.05$.

\section{Swedish Aspen collection RNA-Sequencing data}

The RNA-Seq data used in this study has been described previously in Mahler et al. (2017). It consists of 219 samples distributed among 86 distinct genotypes. The same type of gene expression filtering and adjustment were used in this paper as in Mahler et al.(2017). Genes were required to have an expression variance above 0.05 , and the first nine gene expression principal components were regressed out from the data. This left 22,306 genes for further analysis. The data have been uploaded to the European Nucleotide Archive (ENA) with accession number ERP014886.

\section{Genome wide association mapping}

A total of 4.5 million SNPs were considered for the GWAS, as detailed in Mahler et al. (2017). Best linear unbiased predictors (BLUPs) of the three leaf traits considered (leaf area, indent depth, and circularity) were calculated using the lmer function in the lme4 $\mathrm{R}$ package. The model used was specified in $\mathrm{R}$ as

$\mathrm{y} \sim$ garden + year + block $+(1 \mid$ genotype $)$

Where $\mathrm{y}$ is the phenotype, garden is the common garden where the phenotype was measured, year is the year the phenotype was measured, block is the location of the tree in the common garden, and genotype is the genotype of the sampled tree. A univariate linear mixed model was applied to the data using GEMMA v0.94 (Zhou \& Stephens, 2014) and included days to bud set (Wang et al., 2018) as a covariate in order to account for population structure as well as the built-in estimation of a centred relatedness matrix to control for population structure and relatedness. GEMMA produces different statistics for significance, and in this study, we used $P$-values based on a likelihood ratio test. These $P$-values were consequently BenjaminiHochberg adjusted for multiple testing for each garden and year separately using the p.adjust function in R. To estimate the proportion of variance explained (PVE) by all SNPs for each phenotype, a Bayesian sparse 
linear mixed model (BSLMM) was applied in GEMMA using the Markov chain Monte Carlo (MCMC) method (Zhou, Carbonetto, \& Stephens, 2013). For each of the three traits, MCMC chain length was set to 1000000 steps with the first 250000 discarded as burn-in and thinned to every $100^{\text {th }}$ sample resulting in 10 000 independent draws from the posterior distribution. The median of the posterior distribution of the pve parameter was taken as a point estimate of the proportion of the phenotypic variance explained by all SNPs. To associate genes with SNPs, the v1.0 Populus tremula annotations from the PopGenIE.org web resource was used (Sundell et al., 2015), and any gene within $2 \mathrm{kbp}$ of a SNP were said to be associated with that SNP.

The top 1,000 genes for each of the three traits were selected by ordering the associations by significance and then walking down the list until 1,000 unique genes had been selected.

\section{GWAS gene set enrichment analysis}

The gene set enrichment analysis performed was inspired by Subramanianet al. (2005). The most significant $\mathrm{SNP}$ within $2 \mathrm{Kbp}$ of a gene was used to rank all genes found in the GWA results. For each gene set a running sum was made where the value was increased proportionally to the $P$-value of the most significant association within the gene, if the gene was in the gene set being tested, otherwise the value was decreased. The maximum value in this running sum acted as a test statistic, and 10,000 permutations of the gene ranking was performed, and empirical $P$-values were calculated. The genes in the gene set tested that contributed to the maximum score in the running sum,i.e. genes that occurred before or at the maximum in the running sum, were considered part of the leading-edge subset. The algorithm was implemented in $\mathrm{C}++$ using Rcpp (Eddelbuettel \& Balamuta, 2017) and is available athttps://gist.github.com/maehler/239e18erd9f2b53c792c05f2aca5cebd. Multiple testing correction was performed using the qvalue function in the qvalue $\mathrm{R}$ package. Only gene sets containing more than five genes were considered for the enrichment test. The exponent parameter of the GSEA test was set to 1 for all tests.

\section{Genotype extreme group differential expression}

The top and bottom quartiles of the BLUPs for leaf area, indent depth, and circularity were contrasted in the gene expression data from the SwAsp population (Mahler et al., 2017) using DESeq2 (Love, Huber, \& Anders, 2014). The data were not subset before creating the model, but rather contrasted on the top and bottom quartiles in order to not lose information from samples with intermediate phenotypic values. Genes with an adjusted $P$-value $<0.05$ were considered differentially expressed. Functional enrichment tests were performed as detailed above.

When testing for differences in network centrality among differentially expressed genes, a randomisation scheme was employed in order to assert that there was no circular reasoning behind the connection between DE and network centrality. The sample labels within the top and bottom quartiles for leaf area were shuffled, and differential expression analysis was performed on this new dataset. This was repeated 100 times for leaf area in order to get an indication as to whether DE between random subsets of samples was inherently connected with network centrality.

\section{Plant Material}

We collected root cuttings of diameter 5-10 mm from a wild-growing clonal stand of Populus tremula in northern Sweden (as detailed in Sundell et al. (2017)) on 29th July 2013. We divided the root cuttings into $25 \mathrm{~cm}$ lengths and placed these onto pre-watered potting compost (K-jord, Hasselfors, Orebro, Sweden) in trays and then covered the root sections with $2 \mathrm{~cm}$ of additional compost. The trays were kept damp and placed in a greenhouse with mean day/night temperature of 20/15 degC, humidity 50-70\% and an 18-hour photoperiod. After three weeks, vegetative shoots of approximately $5 \mathrm{~cm}$ height were separated from the root sections and planted into two-litre pots containing potting compost (K-jord, Hasselfors, Orebro, Sweden) and grown in a greenhouse for 12 weeks (24h light, 22 degrees, 50-70 \% humidity). 
Leaf Plastochron Index (LPI; (Erickson \& Michelini, 1957; Larson \& Isebrands, 1971; Meicenheimer, 2014) has been extensively used forPopulus research as a means to sample leaves of equivalent developmental age from replicate plants. We first established that the production of terminal leaves in aspen obeys the assumptions of LPI.

We collected a developmental series of terminal leaves to perform morphological and transcriptional assays. The first fully unfurled leaf was defined as a reference point and was labelled leaf T0. Three leaves above the reference leaf (labelled as T-1, T-2, T-3) and the apical region, containing the shoot apical meristem and the very youngest leaf primordia (labelled T-4) and two leaves below the reference leaf (labelled T1, T2) were sampled from five replicate trees for RNA extraction and from an additional five replicate trees for morphological analyses. Samples collected for RNA extraction were immediately flash frozen in liquid nitrogen and stored at $-80 \operatorname{deg} \mathrm{C}$.

\section{RNA extraction}

We extracted total RNA from terminal leaves above the reference leaf (samples T- 1 through T-4) in the terminal leaf developmental series using the RNAqueous Micro Kit (Life Technologies, Carlsbad, CA, USA) according to the manufacturer's guidelines. For all other terminal leaves (samples T0 through T2) and for all pre-formed leaf samples (first described by Mahler et al. 2017), RNA was isolated using the mirVana Kit (Life Technologies, Carlsbad, CA, USA) according to the manufacturer's guidelines. We could not extract RNA from the smallest terminal leaf samples (samples T-1 through T-4) using the mirVana Kit (Life Technologies, Carlsbad, CA, USA) and we therefore used the RNAqueous Micro Kit (Life Technologies, Carlsbad, CA, USA) for those samples. DNA was removed using the DNA-free ${ }^{\mathrm{TM}}$ DNA removal Kit (Life Technologies, Carlsbad, CA, USA) according to the manufacturer's instructions. RNA purity was measured using a NanoDrop 2000 (Thermo Scientific) and RNA integrity assessed with a Plant RNA Nano Kit using a Bioanalyzer 2000 (Agilent Technologies) using the plant total RNA setting.

\section{Leaf physiognomy}

To enable analysis of leaf size and shape (physiognomy) parameters we sampled a terminal leaf developmental series for which a reference leaf was defined as above. We sampled one leaf younger than the reference leaf (leaf T-1), the reference leaf itself (leaf T0) and five subsequent leaves older than the reference leaf (leaf T1 to leaf T5). Leaves younger than leaf T-1 were not suitable for use in this analysis. As such, the RNA set of samples profiled leaves younger than were represented in this physiognomy developmental series. This developmental series was sampled from five clonally replicated trees.

To obtain images for all leaves we first removed the petioles and subsequently scanned the leaves on a flatbed scanner (Canon LiDE210, New York, USA). Scanned images were saved as 300 dpi colour JPEG files. Leaf shape parameters were then calculated using LAMINA (Bylesjo et al., 2008). Quantified traits included leaf area, length, width, serration number and dimensions in addition to a number of calculated traits such as aspect ratio (length:width) and circularity. Principal components were calculated for use in Table 1.

\section{RNA sequencing data pre-processing}

RNA-Seq of the long-fraction of the extracted total RNA was performed by the Beijing Genome Institute using the Illumina sequencing platform with mRNA assayed using $>20$ million 2 x100 bp paired-end reads per sample. The data are deposited in the European Nucleotide Archive (ENA) as accession PRJEB31491. Protocol details were as presented in (Nystedt et al., 2013).

An in-house pipeline that combines a number of existing tools was used for data processing and expression value calculation (Delhomme et al., 2015): Sequence data quality was assessed using FastQC/0.10.1 (Andrews, n.d.). Sequence reads originating from ribosomal RNAs (rRNA) were identified and removed using SortMeRNA/1.9 (Kopylova, Noe, \& Touzet, 2012) for which libraries rfam-5s, rfam-5.8s, silva-arc-16s, silvabac-16s, silva-euk-18s, silva-arc-23s, silva-bac-23s and silva-euk-28s were used. The sequence data were then 
processed to remove low quality bases or entire reads and adapter contamination was removed using Trimmomatic/0.32 (Bolger, Lohse, \& Usadel, 2014). The trimming parameters used were: SLIDINGWINDOW:5:20 MINLEN:50 while trimming the adapter TruSeq3-PE. After each of rRNA removal and quality filtering the remaining sequence data were assessed again using FastQC/0.10.1. The reads were mapped to the de novo Populus tremula v1.0 genome (pre-release genome available at PopGenIE.org) using STAR/2.4.0f1 (Dobin et al., 2013) with the settings -runThreadN 16, -readFilesCommand zcat, -limitBAMsortRAM, -outQSconversionAdd -31, -outSAMtype BAM SortedByCoordinate, -outSAMstrandField intronMotif, outSAMmapqUnique 254,-outWigType bedGraph, -outFilterMultimapNmax 100,-alignIntronMax 11000, -chimSegmentMin 1, -sjdbGTFfile Potra01-gene-wo-intron.gtf -quantMode TranscriptomeSAM. Alignments to features were counted to enable gene loci expression quantification using HTSeq/0.6.1 (Anders, Pyl, \& Huber, 2014), for which a GFF3 file containing a representative transcript per coding loci was used.

\section{Differential expression inference and functional enrichment analysis}

The gene expression data were first visually assessed by performing a Principal Component Analysis (PCA) and clustered heatmaps using blind variance stabilized data (Lin, Du, Huber, \& Kibbe, 2008). This identified one sample of leaf 4 from the terminal leaf development series as a clear outlier. We therefore excluded this sample from subsequent analysis. Differentially expressed genes (DEGs) between developmental stages were inferred within the $\mathrm{R}$ framework (R Core Team, 2018) using DESeq2 (Love et al., 2014) with a formula including the replicate and developmental stage as a term ( Replicate + Time $)$.

Gene Ontology (Ashburner et al., 2000) functional enrichment (over-representation) of DEGs at $P<0.05$ was analysed using an in-house implementation of the parent-child test (Grossmann, Bauer, Robinson, \& Vingron, 2007) and PFAM domain (Finn et al., 2010) enrichments were calculated using a hypergeometric test.

\section{Gene network inference}

We inferred a gene co-expression network for the terminal leaf RNA-Seq dataset. The expression data were first filtered to only include genes with nonzero expression in at least $\lceil\sqrt{N}\rceil$ samples, which were transformed to homoscedastic, asymptotically $\log 2$ counts using the regularized log transformation as implemented in DESeq2. Ten network inference methods were computed using the Seidr 0.9 toolkit (Schiffthaler, Serrano, Delhomme, \& Street, 2018) - ARACNE, CLR, GENIE3, Narromi, PCor, Pearson, PLSNET, Spearman, SVM and Tigress. For each symmetric edge pair, the one with the higher score was kept in case of nonsymmetrical scoring by the algorithm. The networks were aggregated using the inverse rank product method (Zhong, Allen, Xiao, \& Xie, 2014) and edges were filtered according to the noise corrected backbone (Coscia \& Neffke, 2017) at a sigma of 2.32 (which roughly corresponds to a $P$-value of $1 \%$ ). Network partitions were identified using InfoMap (Rosvall \& Bergstrom, 2008) with default settings. Network comparisons were performed using functions included in Seidr. Graph layout and images were created using Gephi 0.9.2. Node centrality statistics were calculated using Seidr 0.9, except for Kleinberg's Hub and Authority, which was calculated using Gephi 0.9.2.

\section{Results}

We first performed a characterisation of the two heteroblastic leaf forms produced by $P$. tremula. Preformed leaves were orbicular and those produced from the extending shoot apical meristem (terminal) were cordate (Figure 1A, B), which is in agreement with previous observations in poplar (Y. Liu et al., 2015; Russin \& Evert, 1984). Pre-formed leaves were notably thicker $(\sim 2 \mathrm{x})$ than terminal leaves, with contrasting spatial arrangement of cell layers between the two leaf types (Figure 1C, D). Pre-formed leaves had a thicker adaxial epidermal layer followed by two rows of densely packed palisade mesophyll; spongy mesophyll cells were separated by many air spaces and a thick abaxial epidermis. The spongy mesophyll cell layer of terminal leaves had fewer air spaces than in pre-formed leaves (Figure 1C, D). 


\section{Genome wide association mapping identifies complex genetic architecture}

We characterised variation in pre-formed leaf shape within the Swedish Aspen (SwAsp) collection of $P$. tremula genotypes, which was sampled from local populations in Sweden and established in two replicated common garden experiments located in the north (Savar, Umea, 62degN) and south (Ekebo, 56degN) of Sweden (Luquez et al., 2008). We measured three representative leaf physiognomy traits: leaf area, circularity and indent depth (margin/boundary serration) in both common gardens in two years. We observed extensive natural variation in pre-formed leaf shape (Figure 2A), with measured leaf shape traits having high broad sense heritability $\left(H^{2}\right)$ and low sub-population differentiation $\left(Q_{\mathrm{ST}}\right)$ (Table 1). Genetic correlations indicated that a substantial proportion of the heritable variation for each trait is controlled by genetic factors unique to that trait (Figure 2B; Table 1). We additionally tested whether any of the traits correlated significantly to a range of environmental and climatic factors (Table 1). There was no evidence of sub-population differentiation for any of the traits, as indicated by low $Q \mathrm{ST}_{\mathrm{ST}}$ (Table 1) and genotypes did not cluster by sub-population of origin on the basis of the three phenotypic traits (Figure 2D). Similarly, there were no large-effect significant correlations between the traits and environmental and climatic factors (Table 1). Leaf circularity and indent depth had higher $\mathrm{H}^{2}$ and lower Genotype by Environment (GxE) interaction than did leaf area (indicated by comparisons of ANOVA $F$ values; Table 1; Figure S1).

We used the trait values to perform association mapping using genome-wide single nucleotide polymorphism (SNP) markers identified from population-wide resequencing data (Wang et al., 2018). Since there were no statistically significant associations after multiple testing correction for any trait in any of the repeated datasets, we examined whether there was overlap among the top 1,000 SNP associations (rank ordered by $P$-value), indicative of consistency in ranking among the repeated measures. In general, there were few associations in common among the repeated measures, with the majority of the top-ranked associations being unique to each dataset (Figure 2C). In agreement with the lower GxE and higher $\mathrm{H}^{2}$ for leaf circularity and indent depth, these traits displayed greater overlap among the associations than for leaf area. We also calculated Best Linear Unbiased Predictions (BLUPs) from the repeated measures of each trait, but again found no statistically significant associations after multiple testing correction for the BLUPs (Figure S2, Table S1). There was no evidence of substantial inflation due to population stratification (Yang et al., 2011), as indicated by Genomic Control (GC) values ( $\lambda \mathrm{GC} 1.05$ for area, 1.04 for circularity, 1.04 for indent depth) while SNP-based estimates of percentage variance explained (PVE) were relatively high $(0.40 \pm$ s.d. 0.31 for area, $0.63 \pm$ s.d. 0.31 for indent depth, $0.80 \pm$ s.d. 0.28 for circularity), further supporting that higher plasticity of leaf area and that trait variance was under genetic control.

To select candidate genes for these traits, we again rank ordered the SNP associations by $P$-value and selected the top 1,000 genes for each of the traits (Table S2). The majority of associations were unique to each trait (Figure S3), in line with genetic correlations suggesting largely independent genetic control (Figure 2B). We examined the genomic context of SNPs within the top 1,000 gene sets (Figure 3), observing that the highest density of SNPs occurred in regulatory regions (UTRs and flanking regions, which contain the promoter).

\section{Differential gene expression between genotypes with contrasting leaf shape}

We used an existing resource assaying gene expression in flushing leaf buds from the SwAsp collection (Mähler et al., 2017) to examine correlations between gene expression and the leaf physiognomy traits. Although none of these correlations were significant (Figure S4A), we did find significantly Differentially Expressed Genes (DEGs) between sets of genotypes at the population distribution extremes for the three phenotypic measures (Figure 4) including 182 DEGs for area, 203 for circularity and 223 for indent depth (Table S3). As the two shape traits had higher $\mathrm{H}^{2}$, we concentrated more specifically on these in subsequent analyses.

\section{Extensive remodulation of the transcriptome during leaf development}

To characterise the developmental role of our candidate genes, we profiled gene expression using RNA-Seq in a developmental series of terminal leaves from a single genotype. Developmental time accounted for the 
largest proportion of variance in the corresponding gene expression data with the apical region sample being distinctly separated from later developmental stages and with more extensive separation of later stages (Figure 5A). We used these data to perform differential expression analysis (Figure 5B) and to confirm that we observed the expected expression profiles for Gene Ontology (GO) categories (Figure 5C) and homologs of known leaf development regulators (Figure 5D). To be able to examine the relationship to network topology of our GWAS and DEG candidate genes we performed unsupervised network analysis to obtain an unbiased overview of major expression profiles and underlying processes active during leaf development and to identify the most central genes involved in these processes. We calculated a gene co-expression network (Table 2) by aggregating networks from multiple inference algorithms (Marbach et al., 2012). Our analysis involved graph partitioning to define modules (clusters) of genes and subsequent calculation of node centrality statistics (Table 3, Table S4). We identified statistical enrichment of GO and PFAM (Protein family) categories within graph clusters to annotate common processes represented by cluster members (Figure S5 Table S5). We overlaid stage-wise DE results (Table S6) onto the network nodes to visualise network regions or clusters active at each leaf development stage transition and to examine whether most significantly DE genes (DEGs) exhibited an increase or decrease in expression (Figure 6).

To aid community utilisation, we have made the gene expression data and the co-expression network available at http://aspleaf.plantgenie.org and within PopGenIE (Sundell et al., 2015) as the dataset 'AspLeaf'.

\section{Developmental characterisation of population candidate genes}

The majority of the GWAS candidate genes were expressed within the sampled leaf ages but were not included in the developmental network, indicating low levels of connectivity (436 GWAS leaf area genes, 451 circularity genes and 424 indent depth genes were included in the network). None of the genes that were present in the network were highly ranked in terms of network connectivity metrics and none of the network clusters were enriched for these GWAS genes. Furthermore, we performed Gene Set Enrichment Analysis (GSEA) of the GWAS genes using network PageRank score as a measure of network centrality to rank-order genes within the developmental network, finding no significant enrichment (Daub et al., 2013). However, the trend was for GWAS genes being present among low connectivity genes (Figure S6). As such, genes identified by GWAS were clearly not central within the developmental network.

We performed GO enrichment analysis of the GWAS candidate genes, however none had significant enrichment, suggesting that they included genes spanning a diverse range of biological processes and that no particular process was associated with the genetic control of trait variation among these genes. As an alternative to analysing the discrete set of GWAS candidate genes, we used the SNP with the strongest association (lowest $P$-value) within each gene to rank order all genes for each trait. We then performed GSEA using gene sets from GO terms and expression network clusters. A number of GO terms were enriched for the three traits, including "protein phosphorylation" (GO:0006468) for circularity, "sulfur compound metabolic process" (GO:0006790) for indent depth, and "carbohydrate metabolic process" (GO:0005975) for area. Among the network clusters, five were significant at a $5 \%$ false discovery rate. Clusters [1:4], [1:5], and [1:10] were enriched in the leaf area GWAS results and clusters [1:1], [1:4], and [1:5] were enriched for circularity. No cluster was significant for indent depth.

Many of the DEGs between leaf shape extremes were actively regulated during the process of leaf development (Figure 7; Figure S7). There was no significant enrichment of GO terms among these sets of DEGs, however GSEA showed that indent depth GWAS genes were significantly enriched in indent depth DEGs $(q=0.0009)$, that circularity GWAS genes were significantly enriched in circularity DEGs $(q=0.009)$, but that there was no enrichment for area. Examination of the correlations between DEGs and the corresponding phenotypic traits showed that, although no single correlation was significant, correlation values for the DEGs were significantly larger than for non-DEGs (Figure S4B). Two of these DEGs were in common for all three traits (Figure S8) but had relatively low and consistent expression within the leaf development datasets: Potra196739g30199 (ATP synthase subunit C) and Potra003791g32371 (mRNA cap guanine-N7 methyltransferase). Within the terminal development network, 71 area, 83 circularity and 85 indent depth DEGs were present. We 
performed GSEA of the sets of DEGs within the terminal development network (Figure S6), which revealed significant under-representation for genes with low connectivity for all three traits $(P=0.004,0.011$ and 0.001, respectively).

The population gene expression data has previously been utilised to perform expression Quantitative Trait Locus (eQTL) mapping and co-expression network analyses (Mähler et al., 2017). A relatively large proportion of the DEGs between leaf shape extremes were also eGenes (i.e. genes for which an eQTL was present): 80 for area, 78 for circularity, and 83 for indent depth. Out of these genes, 22 for circularity and 35 for indent depth were also part of the subset of genes that contributed to the GWAS GSEA signal. We examined the distribution of network centrality (degree) of DEGs and non-DEGs and GWAS genes within the population co-expression network presented in Mähler et al. (2017) and the developmental network (Figure S9) and performed GSEA to test for a relationship to network connectivity within the population co-expression network (Figure S10). DEGs had significantly lower centrality than non-DEGs, a pattern that we confirmed was specific only to DEGs and not to random gene sets (Figure S9). The GSEA revealed that GWAS genes were significantly enriched among low connectivity genes while DEGs were not, although the trend for DEGs was similar (Figure S10). Furthermore, DEGs displayed signatures of relaxed selective constraint (i.e. lower negative selection), as indicated by Tajima's D values (Figure S11). This pattern was observed for DEGs but not for the top ranked 1,000 GWAS gene sets.

\section{Discussion}

Despite the vast variation in leaf shape within and between species, we still have a rather limited understanding of the gene regulatory network underlying the process of leaf development and of the genetic determinants of leaf shape variation among individuals. There is extensive variation in leaf form within an individual (Figure 1) as well as shape variation of pre-formed leaves among individuals (Figure 2A) in our current study system. We used leaf area, circularity and indent depth as representative physiognomy traits, with leaf shape being under tight genetic control (Table 1). There was no apparent link between leaf shape or size and environment, longitude or latitude (Table 1) and $Q$ st values were low. As such, there was no identifiable adaptive role or clear signature of directional selection for leaf shape within the SwAsp collection. In line with this, and in stark contrast to the date of autumn bud set, which is highly adaptive (Wang et al., 2018), we did not identify any significant SNP associations for the three traits. Given the high $H^{2}$ for leaf circularity and indent depth, this indicates complex genetic architecture with a large number of small effect size polymorphisms contributing to the control of leaf shape variation. As the population size of the SwAsp collection is certainly underpowered to detect such small effect associations, we reasoned that calculated $P$ -values would still be informative for rank ordering the importance of SNP associations. We therefore used this rank ordering to identify the top 1,000 associated genes (i.e. GWAS candidate genes) and examined these further. After normalising for feature length, the highest density of SNPs within the candidate genes was observed in UTR and regulatory (up- and down-stream) regions (Figure 3), suggesting that those SNPs potentially act by influencing gene expression.

We note that these findings are highly concordant with the omnigenic model (Boyle, Li, \& Pritchard, 2017; X. Liu, Li, \& Pritchard, 2019), which posits that a majority of genes contributing to trait variance are not directly biologically connected with the trait of interest (X. Liu et al., 2019). The model states that there is a limited set of genes that directly affect a trait (referred to as core genes), while all other genes with expression variation in the relevant tissue are peripheral to the trait. These peripheral genes can havetrans -acting effects that propagate through the highly connected regulatory network that cause individually small changes in the expression of core genes. While each trans effect is small in isolation, the combined effect of these peripheral trans -acting effects will explain the majority of the variation in core genes and, therefore, in trait variation. It has not been resolved whether core genes defined on the basis of topology within a co-expression network represent core genes as defined by the omnigenic model. It also remains to be explored whether the ability to predict phenotype from gene expression profiles, or co-expression modules, is an effective means of identifying omnigenic core genes. 
We further explored network topology of our identified GWAS candidate genes. A common characteristic of core genes (as defined by the omnigenic model) and of network hubs is that they are under strong selective constraint as natural selection acts strongly against large effect variants (Gouy, Daub, \& Excoffier, 2017; X. Liu et al., 2019; Simons, Bullaughey, Hudson, \& Sella, 2018). To test whether this held up in our system, we focused on a reference genotype to establish a developmental timeline for terminal leaves. As predicted by the model, our GWAS candidate genes were not hubs within the co-expression network constructed from the leaf developmental series.

Our observation that the highest density of top-ranked SNPs was within regulatory regions prompted us to make use of an existing resource of gene expression data assaying flushing buds from the same population (Mähler et al., 2017). We have previously performed eQTL mapping using these data, the results of which also highlighted the importance of SNPs located within regulatory regions in controlling natural variation in transcript abundance (Mähler et al., 2017). We did not identify any genes with significant correlation of gene expression variation among genotypes to variation in our target leaf traits and correlations were of low magnitude (Figure S4). This is, however, expected given the apparently highly polygenic nature of these traits.

In addition to the GWAS candidate genes, we also derived a set of candidate genes based on the population expression data. To this end, we identified a set of genes with the maximum signal strength between expression and phenotype by performing DE analyses between sets of genotypes with the most extreme phenotype values (Figure 4). There was significantly higher correlation for DEGs than non-DEGs and three-way overlap between GWAS, DEGs and eQTL presence provided circumstantial evidence that these genes may influence leaf shape via gene expression variation and that this effect is under genetic control. The sets of DEGs were of lower centrality within the population wide co-expression network (Figure S10) and displayed evidence of weaker negative selection (Figure S11). However, for area and indent depth there was an under-representation of low connectivity genes within the development co-expression network. As such, these candidate genes were under-represented for genes for which changes to expression would be expected to have the lowest impact to the network in general and, by extension, on phenotype. The majority of these genes have no known function in leaf development, but many were actively regulated during leaf development (Figure 7, Figure S7). These results are highly concordant with the omnigenic model, indicating that the DEG analysis potentially identified a set of core trait genes (as defined by the omnigenic model) that have higher effect size on phenotype and higher correlation of expression to phenotype alongside a larger set of genes that are peripheral to the phenotype and that are distributed throughout the developmental co-expression network. Within the GWAS candidate genes there was no such under-representation, suggesting that the DEGs were enriched for genes of higher importance within the development co-expression network and, therefore, of larger impact on phenotype, although this signal is weak. The lack of GO enrichment within the GWAS or DEG candidate gene sets is also congruent with the omnigenic model as the majority of genes contributing to trait heritability are expected to be peripheral and not associated to particular biological processes or directly connected to the focal trait. Taken together our results suggest that leaf shape is an omnigenic trait, with variation resulting from SNPs within regulatory regions that act by causing variation in gene expression. The genes affected are enriched within the periphery of the population co-expression network and are associated with signatures of relaxed selective constraint. There was a lack of evidence for directional selection acting on leaf shape, as indicated by low sub-population differentiation and a lack of any link to environmental variables. This potentially indicates that variation in leaf shape is adaptively neutral within the morphospace represented among the sampled genotypes, although there are other plausible explanations congruent with the results. However, neutrality does appear to be an harmonious interpretation as allele frequencies of SNPs amoung the GWAS candidate genes reflected those of all SNPs globally and of the 1000 lowest ranked SNPs. There was, therefore, no clear indication of balancing or stabilising selection or of extensive purifying selection. Of note, the pattern was not the same for area, for which SNPs within GWAS candidate genes showed a skew towards lower allele frequencies. This suggests that there may be contrasting selection pressures acting on leaf size and shape, with leaf size being subjected to stronger purifying selection, although the signal was weak, and this was not reflected in the distribution of Tajima's D (Figure S11). 
Similar approaches have been used to identify and prioritise candidate genes in maize, with small numbers of those candidates confirmed to influence leaf characteristics in transgenic lines (Baute et al., 2015, 2016; Schaefer et al., 2018). A distinct difference in maize is the clear separation of cell proliferation and subsequent expansion into defined zones that can easily be sampled separately. This sampling approach increases signal strength for associating gene expression to division and expansion processes, which were mixed within single samples within our current study. In maize there are reported loci of higher effect size influencing leaf development and phenotype, possibly as those traits are correlated to general yield characteristics (Baute et al., 2016) and have therefore been targets of artificial selection. A similar study in apple reported a small number of significant associations for leaf shape traits but concluded that those were false-positives (Crouch et al., 2018) and a study in sweet potato used a similar DEG approach to identify genes associated with variation in leaf form (Gupta et al., 2019).

The analysis of leaf shape has many parallels with that of face shape in humans, for which initial GWAS of basic parameters (such as length:width) yielded few to no significant associations. However, analyses that defined more specific sub-features have yielded substantially more informative associations (Crouch et al., 2018). In aspen, there is similarly considerable scope to improve phenotype GWAS results through improved software tools to deconvolute more specific features of leaf shape, such as the angle of veins or of specific regions of the leaf, such as the base and tip. Each of these features is likely under specific molecular control that exhibits genetic variation among individuals and that are not captured by the larger scale features, such as overall leaf circularity, employed in our current study. The fact that such sub-features of leaf shape are under local, spatially defined control and that growth distribution across the leaf lamina controls final leaf shape and venation patterning (Runions, Tsiantis, \& Prusinkiewicz, 2017) may also explain the low correlation between leaf shape traits and gene expression in the whole-bud samples used to generate the gene expression values for the eQTL mapping results that we considered here. Furthermore, the use of a single developmental snapshot for such analysis is limiting as causal variation in expression could occur at any point during the developmental program.

We integrated developmental gene expression profiling, GWAS, population-wide gene expression data and population genetics to define the genetic architecture of leaf shape variation in aspen. We show that leaf shape variation is a highly complex trait likely determined by small-effect variations in gene expression caused by numerous small-effect size SNPs in regulatory regions. Genes with evidence of association to variation in leaf shape were peripheral within a population-wide gene co-expression network, were not hubs within the leaf developmental network and displayed signatures of relaxed selection. We therefore suggest that leaf shape is an omnigenic trait. Combined with low sub-population differentiation and a lack of correlation to climatic or environmental variables, we suggest that variation in leaf shape within the morphospace represented within the SwAsp collection may be neutral. We believe that this integrated approach is a pragmatic strategy for identifying candidate genes and disentangling the genetic basis of complex traits, such as leaf shape variation in aspen, which have no apparent evidence of being adaptive and for which no significant associations are identified using association mapping.

\section{Acknowledgements}

NRS, BT and KMR were supported by the Trees and Crops for the Future (TC4F) project. NM, BS and CM were supported by the Knut and Alice Wallenberg foundation and the VINNOVA UPSC Centre for Forest Biotechnology. This work was supported by the Trygger Foundation (\#CTS 12:471) and Gunnar och Ruth Björkmans fond för Norrländsk Botanisk Forskning. The authors acknowledge support from the Umeå Plant Science Centre Bioinformatics platform (UPSCb), the Science for Life Laboratory (SciLifeLab)and the National Genomics Infrastructure (NGI). Computations were performed on resources provided by SNIC through Uppsala Multidisciplinary Center for Advanced Computational Science (UPPMAX). We thank Skogforsk for hosting the SwAsp collection common gardens. 


\section{References}

Aida, M., Ishida, T., Fukaki, H., Fujisawa, H., \& Tasaka, M. (1997). Genes involved in organ separation in Arabidopsis: an analysis of the cup-shaped cotyledon mutant. The Plant Cell , 9 (6), 841-857. doi: 10.1105/tpc.9.6.841

Anders, S., Pyl, P. T., \& Huber, W. (2014). HTSeq - A Python framework to work with high-throughput sequencing data. Bioinformatics (Oxford, England) , 31 (2), 166-169. doi: 10.1101/002824

Andres, R. J., Coneva, V., Frank, M. H., Tuttle, J. R., Samayoa, L. F., Han, S.-W., ... Kuraparthy, V. (2017). Modifications to a LATE MERISTEM IDENTITY1 gene are responsible for the major leaf shapes of Upland cotton ( Gossypium hirsutum L.). Proceedings of the National Academy of Sciences , 114 (1), E57-E66. doi: 10.1073/pnas.1613593114

Andrews, S. (n.d.). FastQC. Retrieved from http://www.bioinformatics.babraham.ac.uk/projects/fastqc/ website: http://www.bioinformatics.babraham.ac.uk/projects/fastqc/

Ashburner, M., Ball, C., Blake, J., Botstein, D., Butler, H., Cherry, J., .. Sherlock, G. (2000). Gene ontology: tool for the unification of biology. The Gene Ontology Consortium. Nature Genetics , 25 (1), 25-29. doi: $10.1038 / 75556$

Barnes, B. V. (1969). Natural variation and delineation of clones of Populus tremuloides and P. grandidentata in northern lower Michigan. Silvae Genetica , 18 , 130-142.

Barnes, B. V. (1975). Phenotypic variation of Trembling Aspen in western North America. Forest Science , 21 (3), 319-328.

Baute, J., Herman, D., Coppens, F., De Block, J., Slabbinck, B., Dell'Acqua, M., ... Inzé, D. (2015). Correlation analysis of the transcriptome of growing leaves with mature leaf parameters in a maize RIL population. Genome Biology , 16 (1), 168. doi: 10.1186/s13059-015-0735-9

Baute, J., Herman, D., Coppens, F., De Block, J., Slabbinck, B., Dell'Acqua, M., ... Inzé, D. (2016). Combined Large-Scale Phenotyping and Transcriptomics in Maize Reveals a Robust Growth Regulatory Network. Plant Physiology , 170 (3), 1848-1867. doi: 10.1104/pp.15.01883

Bolger, A. M., Lohse, M., \& Usadel, B. (2014). Trimmomatic: a flexible trimmer for Illumina sequence data. Bioinformatics ,30 (15), 2114-2120. doi: 10.1093/bioinformatics/btu170

Boyle, E. A., Li, Y. I., \& Pritchard, J. K. (2017). An Expanded View of Complex Traits: From Polygenic to Omnigenic. Cell , 169 (7), 1177-1186. doi: 10.1016/J.CELL.2017.05.038

Bylesjö, M., Segura, V., Soolanayakanahally, R. Y., Rae, A. M., Trygg, J., Gustafsson, P., .. Street, N. R. (2008). LAMINA: a tool for rapid quantification of leaf size and shape parameters. BMC Plant Biology , 8 (1), 82. doi: 10.1186/1471-2229-8-82

Cheng, X., Peng, J., Ma, J., Tang, Y., Chen, R., Mysore, K. S., \& Wen, J. (2012). NO APICAL MERISTEM (MtNAM) regulates floral organ identity and lateral organ separation in Medicago truncatula. New Phytologist , 195 (1), 71-84. doi: 10.1111/j.1469-8137.2012.04147.x

Chitwood, D. H. H., \& Sinha, N. R. R. (2016). Evolutionary and Environmental Forces Sculpting Leaf Development. Current Biology ,26 (7), R297-R306. doi: 10.1016/j.cub.2016.02.033

Chitwood, D. H., Kumar, R., Headland, L. R., Ranjan, A., Covington, M. F., Ichihashi, Y., .. Sinha, N. R. (2013). A Quantitative Genetic Basis for Leaf Morphology in a Set of Precisely Defined Tomato Introgression Lines. The Plant Cell , 25 (7), 2465-2481. doi: 10.1105/tpc.113.112391

Chitwood, D. H., Maloof, J. N., \& Sinha, N. R. (2013). Dynamic transcriptomic profiles between tomato and a wild relative reflect distinct developmental architectures. Plant Physiology ,162 (2), 537-552. doi: $10.1104 /$ pp.112.213546 
Chitwood, D. H., Ranjan, A., Kumar, R., Ichihashi, Y., Zumstein, K., Headland, L. R., .. Sinha, N. R. (2014). Resolving Distinct Genetic Regulators of Tomato Leaf Shape within a Heteroblastic and Ontogenetic Context. The Plant Cell , 26 (9), 3616-3629. doi: 10.1105/tpc.114.130112

Chitwood, D. H., Ranjan, A., Martinez, C. C., Headland, L. R., Thiem, T., Kumar, R., ... Sinha, N. R. (2014). A modern ampelography: a genetic basis for leaf shape and venation patterning in grape.Plant Physiology , 164 (1), 259-272. doi: 10.1104/pp.113.229708

Clark, S. E. (1997). Organ Formation at the Vegetative Shoot Meristem. The Plant Cell , 9 (7), 1067-1076. doi: $10.1105 /$ tpc.9.7.1067

Clauw, P., Coppens, F., Korte, A., Herman, D., Slabbinck, B., Dhondt, S., .. Inzé, D. (2016). Leaf Growth Response to Mild Drought: Natural Variation in Arabidopsis Sheds Light on Trait Architecture. The Plant Cell , 28 (10), 2417-2434. doi: 10.1105/tpc.16.00483

Cleland, R. E. (2001). Unlocking the mysteries of leaf primordia formation. Proceedings of the National Academy of Sciences of the United States of America , 98 (20), 10981-10982. doi: 10.1073/pnas.211443498

Coscia, M., \& Neffke, F. (2017). Network Backboning with Noisy Data.ArXiv, arXiv:1701 .

Cox, S. E. (2005). Elevational gradient of neoformed shoot growth inPopulus tremuloides . Canadian Journal of Botany ,83 (10), 1340-1344. doi: 10.1139/b05-103

Critchfield, W. B. (1960). Leaf Dimorphism in Populus Trichocarpa.American Journal of Botany , 47 (8), 699. doi: $10.2307 / 2439521$

Crouch, D. J. M., Winney, B., Koppen, W. P., Christmas, W. J., Hutnik, K., Day, T., .. Bodmer, W. F. (2018). Genetics of the human face: Identification of large-effect single gene variants. Proceedings of the National Academy of Sciences of the United States of America ,115 (4), E676-E685. doi: 10.1073/pnas.1708207114

Curtis, J. D., \& Lersten, N. R. (1978). Heterophylly in Populus grandidentata (Salicaceae) with Emphasis on Resin Glands and Extrafloral Nectaries. American Journal of Botany , 65 (9), 1003. doi: 10.2307/2442687

Czesnick, H., \& Lenhard, M. (2015). Size control in plants-lessons from leaves and flowers. Cold Spring Harbor Perspectives in Biology , 7 (8), a019190. doi: 10.1101/cshperspect.a019190

Daub, J. T., Hofer, T., Cutivet, E., Dupanloup, I., Quintana-Murci, L., Robinson-Rechavi, M., \& Excoffier, L. (2013). Evidence for polygenic adaptation to pathogens in the human genome. Molecular Biology and Evolution , 30 (7), 1544-1558. doi: 10.1093/molbev/mst080

Delhomme, N., Mähler, N., Schiffthaler, B., Sundell, D., Mannapperuma, C., Hvidsten, T. R., ... Street, N. R. (2015). Guidelines for RNA-Seq data analysis. Retrieved March 13, 2016, from Epigenesis website: http://www.epigenesys.eu/en/protocols/bio-informatics/1283-guidelines-for-rna-seq-data-analysis

Dobin, A., Davis, C. A., Schlesinger, F., Drenkow, J., Zaleski, C., Jha, S., .. Gingeras, T. R. (2013). STAR: ultrafast universal RNA-seq aligner. Bioinformatics (Oxford, England) , 29 (1), 15-21. doi: 10.1093/bioinformatics/bts635

Drost, D. R., Puranik, S., Novaes, E., Novaes, C. R. D. B., Dervinis, C., Gailing, O., \& Kirst, M. (2015). Genetical genomics of Populus leaf shape variation. BMC Plant Biology , 15 , 166. doi: 10.1186/s12870-0150557-7

Du, J., Mansfield, S. D., \& Groover, A. T. (2009). The Populus Homeobox Gene ARBORKNOX2 Regulates Cell Differentiation During Secondary Growth.The Plant Journal : For Cell and Molecular Biology ,60 (6), 1000-1014. doi: 10.1111/j.1365-313X.2009.04017.x

Eddelbuettel, D., \& Balamuta, J. J. (2017). Extending R with C++: A Brief Introduction to Rcpp. PeerJ Preprints , 5 , e3188v1. doi: 10.7287/peerj.preprints.3188v1 
Erickson, R. O., \& Michelini, F. J. (1957). The Plastochron Index.American Journal of Botany , 44 (4), $297-305$.

Finn, R., Mistry, J., Tate, J., Coggill, P., Heger, A., Pollington, J., .. Bateman, A. (2010). The Pfam protein families database.Nucleic Acids Research , 38 (suppl 1), D211-D222. doi: doi: 10.1093/nar/gkp985

Fulop, D., Ranjan, A., Ofner, I., Covington, M. F., Chitwood, D. H., West, D., .. Sinha, N. R. (2016). A New Advanced Backcross Tomato Population Enables High Resolution Leaf QTL Mapping and Gene Identification. G3 (Bethesda, Md.) , 6 (10), 3169-3184. doi: 10.1534/g3.116.030536

Gouy, A., Daub, J. T., \& Excoffier, L. (2017). Detecting gene subnetworks under selection in biological pathways. Nucleic Acids Research , 45 (16). doi: 10.1093/nar/gkx626

Grossmann, S., Bauer, S., Robinson, P. N., \& Vingron, M. (2007). Improved detection of overrepresentation of Gene-Ontology annotations with parent child analysis. Bioinformatics , 23 (22), 3024-3031. doi: 10.1093/bioinformatics/btm440

Gupta, S., Rosenthal, D. M., Stinchcombe, J. R., \& Baucom, R. S. (2019). The remarkable morphological diversity of leaf shape in sweet potato ( Ipomoea batatas ): the influence of genetics, environment, and GxE. New Phytologist , nph.16286. doi: 10.1111/nph.16286

Ichihashi, Y., Aguilar-Martinez, J. A., Farhi, M., Chitwood, D. H., Kumar, R., Millon, L. V, .. Sinha, N. R. (2014). Evolutionary developmental transcriptomics reveals a gene network module regulating interspecific diversity in plant leaf shape. Proceedings of the National Academy of Sciences of the United States of America ,111 (25), E2616-21. doi: 10.1073/pnas.1402835111

Kopylova, E., Noe, L., \& Touzet, H. (2012). SortMeRNA: fast and accurate filtering of ribosomal RNAs in metatranscriptomic data.Bioinformatics (Oxford, England) , 28 (24), 3211-3217. doi: 10.1093/bioinformatics/bts611

Larson, P. R., \& Isebrands, J. G. (1971). The Plastochron Index as Applied to Developmental Studies of Cottonwood. Canadian Journal of Forest Research , 1 (1), 1-11.

Lin, S. M., Du, P., Huber, W., \& Kibbe, W. A. (2008). Model-based variance-stabilizing transformation for Illumina microarray data.Nucleic Acids Research , 36 (2), e11-e11. doi: 10.1093/nar/gkm1075

Lindtke, D., Gonzalez-Martinez, S. C., Macaya-Sanz, D., \& Lexer, C. (2013). Admixture mapping of quantitative traits in Populus hybrid zones: power and limitations. Heredity , 111 (6), 474-485. doi: 10.1038/hdy.2013.69

Liu, X., Li, Y. I., \& Pritchard, J. K. (2019). Trans Effects on Gene Expression Can Drive Omnigenic Inheritance. Cell , 177 (4), 1022-1034.e6. doi: 10.1016/j.cell.2019.04.014

Liu, Y., Li, X., Chen, G., Li, M., Liu, M., \& Liu, D. (2015). Epidermal Micromorphology and Mesophyll Structure of Populus euphratica Heteromorphic Leaves at Different Development Stages. PloS One ,10 (9), e0137701. doi: 10.1371/journal.pone.0137701

Love, M. I., Huber, W., \& Anders, S. (2014). Moderated estimation of fold change and dispersion for RNA-seq data with DESeq2. Genome Biology , 15 (12), 550. doi: 10.1186/s13059-014-0550-8

Luquez, V., Hall, D., Albrectsen, B. R., Karlsson, J., Ingvarsson, P., \& Jansson, S. (2008). Natural phenological variation in aspen (Populus tremula): the SwAsp collection. Tree Genetics 85 Genomes ,4 (2), 279-292. doi: 10.1007/s11295-007-0108-y

Mahler, N., Wang, J., Terebieniec, B. K. B. K., Ingvarsson, P. K. P. K., Street, N. R. N. R., \& Hvidsten, T. R. (2017). Gene co-expression network connectivity is an important determinant of selective constraint. PLOS Genetics , 13 (4), e1006402. doi: 10.1371/journal.pgen.1006402 
Marbach, D., Costello, J. C., Kuffner, R., Vega, N. N. M. N., Prill, R. J., Camacho, D. M., .. Stolovitzky, G. (2012). Wisdom of crowds for robust gene network inference. Nature Methods , 9 (8), 796-804. doi: $10.1038 /$ nmeth.2016

Meicenheimer, R. D. (2014). The plastochron index: Still useful after nearly six decades. American Journal of Botany , 101 (11), 1821-1835. doi: 10.3732/ajb.1400305

Moon, J., \& Hake, S. (2011). How a leaf gets its shape. Current Opinion in Plant Biology , 14 (1), 24-30. doi: $10.1016 / J . P B I .2010 .08 .012$

Nystedt, B. B., Street, N. R., Wetterbom, A., Zuccolo, A., Lin, Y.-C., Scofield, D. G., ... Jansson, S. (2013). The Norway spruce genome sequence and conifer genome evolution. Nature , 497 (7451), 579-584. doi: $10.1038 /$ nature12211

Peppe, D. J., Royer, D. L., Cariglino, B., Oliver, S. Y., Newman, S., Leight, E., .. Wright, I. J. (2011). Sensitivity of leaf size and shape to climate: global patterns and paleoclimatic applications. New Phytologist , 190 (3), 724-739. doi: 10.1111/j.1469-8137.2010.03615.x

R Core Team. (2018). R: A language and environment for statistical computing (R. F. for S. Computing, Ed.). Retrieved from http://www.r-project.org

Rae, A. M., Ferris, R., Tallis, M. J., \& Taylor, G. (2006). Elucidating genomic regions determining enhanced leaf growth and delayed senescence in elevated CO2. Plant, Cell and Environment, 29 (9), 1730-1741. doi: 10.1111/j.1365-3040.2006.01545.x

Ranjan, A., Budke, J. M., Rowland, S. D., Chitwood, D. H., Kumar, R., Carriedo, L., ... Sinha, N. R. (2016). eQTL Regulating Transcript Levels Associated with Diverse Biological Processes in Tomato.Plant Physiology , 172 (1), 328-340. doi: 10.1104/pp.16.00289

Ratke, C., Terebieniec, B. K., Winestrand, S., Derba-Maceluch, M., Grahn, T., Schiffthaler, B., ... Mellerowicz, E. J. (2018). Downregulating aspen xylan biosynthetic GT43 genes in developing wood stimulates growth via reprograming of the transcriptome. New Phytologist , 219 (1), 230-245. doi: 10.1111/nph. 15160

Rosvall, M., \& Bergstrom, C. T. (2008). Maps of random walks on complex networks reveal community structure. Proceedings of the National Academy of Sciences of the United States of America, 105 (4), 1118-1123. doi: 10.1073/pnas.0706851105

Rottmann, W. H., Meilan, R., Sheppard, L. A., Brunner, A. M., Skinner, J. S., Ma, C., ... Strauss, S. H. (2000). Diverse effects of overexpression of LEAFY and PTLF, a poplar (Populus) homolog of LEAFY/FLORICAULA, in transgenic poplar and Arabidopsis. Plant $J$,22 (3), 235-245. doi: 10.1046/j.1365-313x.2000.00734.x

Royer, D. L., McElwain, J. C., Adams, J. M., \& Wilf, P. (2008). Sensitivity of leaf size and shape to climate within Acer rubrumand Quercus kelloggii . New Phytologist , 179 (3), 808-817. doi: 10.1111/j.14698137.2008.02496.x

Royer, D. L., Wilf, P., Janesko, D. A., Kowalski, E. A., \& Dilcher, D. L. (2005). Correlations of climate and plant ecology to leaf size and shape: potential proxies for the fossil record. Am. J. Bot. ,92 (7), 1141-1151. doi: $10.3732 /$ ajb.92.7.1141

Runions, A., Tsiantis, M., \& Prusinkiewicz, P. (2017). A common developmental program can produce diverse leaf shapes. New Phytologist, 216 (2), 401-418. doi: 10.1111/nph.14449

Russin, W. A., \& Evert, R. F. (1984). Studies on the Leaf of Populus deltoides (Salicaceae): Morphology and Anatomy. American Journal of Botany, 71 (10), 1398. doi: 10.2307/2443707

Schaefer, R., Michno, J.-M., Jeffers, J., Hoekenga, O. A., Dilkes, B. P., Baxter, I. R., \& Myers, C. (2018). Integrating co-expression networks with GWAS to prioritize causal genes in maize. The Plant Cell, 
tpc.00299.2018. doi: 10.1105/tpc.18.00299

Schiffthaler, B., Serrano, A., Delhomme, N., \& Street, N. R. (2018). Seidr: A toolkit for calculation of crowd networks. BioRxiv , 250696. doi: 10.1101/250696

Simons, Y. B., Bullaughey, K., Hudson, R. R., \& Sella, G. (2018). A population genetic interpretation of GWAS findings for human quantitative traits. PLoS Biology , 16 (3). doi: 10.1371/journal.pbio.2002985

Sluis, A., \& Hake, S. (2015). Organogenesis in plants: initiation and elaboration of leaves. Trends in Genetics : TIG , 31 (6), 300-306. doi: 10.1016/j.tig.2015.04.004

Souer, E., van Houwelingen, A., Kloos, D., Mol, J., \& Koes, R. (1996). The no apical meristem gene of Petunia is required for pattern formation in embryos and flowers and is expressed at meristem and primordia boundaries. Cell , 85 (2), 159-170. doi: 10.1016/S0092-8674(00)81093-4

Stettler, R. F., \& Bradshaw Jr, H. D. (1996). Evolution, Genetics, and Genetic Manipulation. In R. . Stettler, H. D. Bradshaw Jr, P. Heilman, \& T. Hinckley (Eds.), Biology of Populus and its implications for management and conservation (pp. 1-6). Ottawa: NRC Research Press.

Street, N. R., Jansson, S., \& Hvidsten, T. R. (2011). A systems biology model of the regulatory network in Populus leaves reveals interacting regulators and conserved regulation. BMC Plant Biology ,11 (1), 13. doi: 10.1186/1471-2229-11-13

Street, N. R., Sjodin, A., Bylesjo, M., Gustafsson, P., Trygg, J., \& Jansson, S. (2008). A cross-species transcriptomics approach to identify genes involved in leaf development. BMC Genomics , 9 (1), 589. doi: 10.1186/1471-2164-9-589

Subramanian, A., Tamayo, P., Mootha, V. K., Mukherjee, S., Ebert, B. L., Gillette, M. A., ... Mesirov, J. P. (2005). Gene set enrichment analysis: a knowledge-based approach for interpreting genome-wide expression profiles. Proceedings of the National Academy of Sciences of the United States of America , 102 (43), 15545-15550. doi: 10.1073/pnas.0506580102

Sundell, D., Mannapperuma, C., Netotea, S., Delhomme, N., Lin, Y.-C. Y.-C., Sjodin, A., ... Street, N. R. N. R. (2015). The Plant Genome Integrative Explorer Resource: PlantGenIE.org. New Phytologist, 208 (4), 1149-1156. doi: 10.1111/nph.13557

Sundell, D., Street, N. R. N. R., Kumar, M., Mellerowicz, E. J. E. J., Kucukoglu, M., Johnsson, C., .. Hvidsten, T. R. (2017). AspWood: High-Spatial-Resolution Transcriptome Profiles Reveal Uncharacterized Modularity of Wood Formation in Populus tremula. The Plant Cell ,29 (7), 1585-1604. doi: $10.1105 /$ tpc. 17.00153

Traiser, C., Klotz, S., Uhl, D., \& Mosbrugger, V. (2005). Environmental signals from leaves - a physiognomic analysis of European vegetation.New Phytologist , 166 (2), 465-484. doi: 10.1111/j.1469-8137.2005.01316.x

Tsuge, T., Tsukaya, H., \& Uchimiya, H. (1996). Two independent and polarized processes of cell elongation regulate leaf blade expansion in Arabidopsis thaliana (L.) Heynh. Development, 122 (5), 1589-1600.

Tsukaya, H. (2005). Leaf shape: genetic controls and environmental factors. The International Journal of Developmental Biology ,49 (5-6), 547-555. doi: 10.1387/ijdb.041921ht

Vroemen, C. W., Mordhorst, A. P., Albrecht, C., Kwaaitaal, M. A. C. J., \& de Vries, S. C. (2003). The CUP-SHAPED COTYLEDON3 gene is required for boundary and shoot meristem formation in Arabidopsis. The Plant Cell , 15 (7), 1563-1577. doi: 10.1105/TPC.012203

Wang, J., Ding, J., Tan, B., Robinson, K. M. K. M., Michelson, I. H. I. H., Johansson, A., .. Ingvarsson, P. K. P. K. (2018). A major locus controls local adaptation and adaptive life history variation in a perennial plant. Genome Biology, 19 (1), 72. doi: 10.1186/s13059-018-1444-y 
Wu, R., Bradshaw, H. D. H., Stettler, R. F. R., Jr., \& Stettler, R. F. R. (1997). Molecular genetics of growth and development in Populus (Salicaceae). v. mapping quantitative trait loci affecting leaf variation. American Journal of Botany , 84 (2), 143-153. doi: 10.2307/2446076

Xiao, D., Wang, H., Basnet, R. K., Zhao, J., Lin, K., Hou, X., \& Bonnema, G. (2014). Genetic Dissection of Leaf Development in Brassica rapa Using a Genetical Genomics Approach. PLANT PHYSIOLOGY,164 (3), 1309-1325. doi: 10.1104/pp.113.227348

Yang, J., Weedon, M. N., Purcell, S., Lettre, G., Estrada, K., Willer, C. J., .. Visscher, P. M. (2011). Genomic inflation factors under polygenic inheritance. European Journal of Human Genetics ,19 (7), 807812. doi: 10.1038/ejhg.2011.39

Zhong, R., Allen, J. D., Xiao, G., \& Xie, Y. (2014). Ensemble-Based Network Aggregation Improves the Accuracy of Gene Network Reconstruction. PLoS ONE , 9 (11), e106319. doi: 10.1371/journal.pone.0106319

Zhou, X., Carbonetto, P., \& Stephens, M. (2013). Polygenic Modeling with Bayesian Sparse Linear Mixed Models. PLoS Genetics ,9 (2), e1003264. doi: 10.1371/journal.pgen.1003264

Zhou, X., \& Stephens, M. (2014). Efficient multivariate linear mixed model algorithms for genome-wide association studies. Nature Methods , 11 (4), 407-409. doi: 10.1038/nmeth.2848

\section{Data Accessibility Statement}

The data are deposited in the European Nucleotide Archive (ENA) as accession PRJEB31491.

\section{Author contributions}

Planned and designed the research: NRS

Performed experiments: NM, BS, KMR, BKT

Contributed resources: NRS, SJ

Contributed analysis ideas: MV, MESB, TRH,

Analyzed data: NM, BS, KMR, BKT, CM

Wrote the paper: NRS with contributions from all authors

Table 1 . Broad sense heritability $\left(\mathrm{H}^{2}\right)$, population differentiation $\left(\mathrm{Q}_{\mathrm{ST}}\right)$, Genotype by Environment interaction $(\mathrm{GxE})$ and environmental correlations for leaf circularity, indent depth and area. Phenotypes were measured in two years (2008 and 2011) and two common gardens (Ekebo in southern Sweden and Savar in northern Sweden). GxE interactions for each phenotype were tested using an ANOVA model with garden, year and garden $\mathrm{x}$ year as independent variables, and phenotype as the dependent variable. The $F$ ratio and $P$-value for the garden:year interaction are reported. Figure S1 details comparisons for each year/garden combination.

\begin{tabular}{lllll}
\hline & Garden/Year & Circularity & Indent depth & Area \\
\hline $\mathrm{H}^{2}(95 \%$ C.I. $)$ & Ekebo 2008 & $0.71(0.64-0.78)$ & $0.60(0.51-0.69)$ & $0.40(0.30-0.51)$ \\
& Ekebo 2011 & $0.65(0.56-0.72)$ & $0.72(0.65-0.79)$ & $0.61(0.52-0.69)$ \\
& Sävar 2008 & $0.75(0.66-0.82)$ & $0.62(0.51-0.72)$ & $0.24(0.12-0.38)$ \\
& Sävar 2011 & $0.71(0.63-0.78)$ & $0.64(0.55-0.73)$ & $0.27(0.16-0.29)$ \\
QST (95\% C.I.) $)$ & Ekebo 2008 & $0.16(0.07-0.37)$ & $0.03(0.00-0.12)$ & $0.09(0.03-0.25)$ \\
& Ekebo 2011 & $0.04(0.01-0.15)$ & $0.02(0.02-0.20)$ & $0.12(0.05-0.31)$ \\
& Sävar 2008 & $0.20(0.09-0.45)$ & $0.09(0.03-0.28)$ & $0.08(0.02-0.25)$ \\
& Sävar 2011 & $0.11(0.04-0.30)$ & $0.05(0.01-0.18)$ & $0.06(0.01-0.20)$
\end{tabular}




\begin{tabular}{lllll}
\hline & Garden/Year & Circularity & Indent depth & Area \\
\hline GxE ANOVA (F) & & 5.309 & 21.669 & 231.0 \\
GxE ANOVA (P) & & 0.0213 & $3.51 \mathrm{e}-06$ & $<2 \mathrm{e}-16$ \\
Latitude R $^{2}$ & Ekebo 2008 & 0.031 & 0.031 & 0.059 \\
& Ekebo 2011 & -0.008 & 0.034 & 0.069 \\
& Sävar 2008 & -0.006 & 0.051 & 0.006 \\
Longitude R ${ }^{2}$ & Sävar 2011 & 0.036 & -0.004 & -0.002 \\
& Ekebo 2008 & 0.021 & -0.009 & 0.009 \\
& Ekebo 2011 & -0.009 & -0.002 & 0.017 \\
Elevation R ${ }^{2}$ & Sävar 2008 & -0.008 & -0.002 & -0.001 \\
& Sävar 2011 & 0.018 & 0.004 & 0.001 \\
& Ekebo 2008 & -0.007 & 0.017 & 0.010 \\
Precipitation $\mathrm{R}^{2}$ & Ekebo 2011 & -0.009 & 0.049 & 0.046 \\
& Sävar 2008 & 0.008 & 0.006 & -0.006 \\
& Sävar 2011 & 0.008 & -0.007 & -0.009 \\
& Ekebo 2008 & 0.024 & -0.008 & 0.057 \\
& Ekebo 2011 & -0.007 & -0.008 & 0.011 \\
& Sävar 2008 & 0.017 & -0.009 & -0.009 \\
& Sävar 2011 & 0.031 & 0.018 & -0.008 \\
\hline
\end{tabular}

Table 2. Summary statistics for networks created from gene expression data.

\begin{tabular}{ll}
\hline Statistic & Terminal network \\
Number of nodes & 14419 \\
Number of edges & 160414 \\
Connected components & 325 \\
Global clustering coefficient & 0.293769 \\
Scale free fit & 0.869421 \\
Average degree & 22.2504 \\
Average weighted degree & 6.95192 \\
Network diameter (largest component) & 10.0627 \\
Average path length & 1.31698 \\
\hline
\end{tabular}

Table 3. Genes with high gene co-expression network centrality measures in the terminal leaf developmental series. Centrality measures are PageRank (PR), Betweenness centrality (BT), Eigenvector centrality (EV), Katz centrality (KT) and Kleinberg's authority (AT). The top five genes for each network metric are detailed. Gene descriptions and bestArabidopsis thaliana BLASTp hits were obtained from PlantGenIE. A. thaliana homologs with reported leaf or whole plant phenotypes in the RARGE (RIKEN Arabidopsis Genome Encyclopedia) database are indicated in bold.

\begin{tabular}{|c|c|c|c|c|c|}
\hline Gene & Description & ATG homolog & $\mathrm{PR}$ & BT & EV \\
\hline Potra000252g00987 & ADP-ribosylation factor & AT1G10630 & & & \\
\hline Potra000353g01326 & histidine kinase 3 & AT1G27320 & & $\mathrm{x}$ & \\
\hline Potra000353g01329 & binding transcription activator & AT1G67310 & & $\mathrm{x}$ & \\
\hline Potra000380g01682 & Ribosomal protein S27 & AT3G61110 & & $\mathrm{x}$ & \\
\hline Potra000389g01777 & homeobox protein knotted (KNAT6) & AT1G23380 & $\mathrm{x}$ & & \\
\hline Potra000404g01918 & ATP synthase subunit epsilon, mitochondrial & AT1G51650 & & & $\mathrm{x}$ \\
\hline Potra001053g08992 & probable linoleate 9S-lipoxygenase & AT1G55020 & & $\mathrm{x}$ & \\
\hline Potra001073g09248 & acid-amido synthetase GH3.6 & AT5G54510 & $\mathrm{x}$ & & \\
\hline
\end{tabular}




\begin{tabular}{|c|c|c|c|c|c|}
\hline Gene & Description & ATG homolog & $\mathrm{PR}$ & $\mathrm{BT}$ & $\mathrm{EV}$ \\
\hline Potra001485g12400 & homeobox protein knotted (KNAT1) & AT4G08150 & $\mathrm{x}$ & & \\
\hline Potra001534g12731 & diphosphooligosaccharide-protein glycosyltransferase subunit 4A & AT3G12587 & & & $\mathrm{x}$ \\
\hline Potra001842g14793 & Ubiquitin-conjugating enzyme & AT1G78870 & & & $\mathrm{x}$ \\
\hline Potra002003g15717 & homeobox protein STM & AT1G62360 & $\mathrm{x}$ & & \\
\hline Potra003440g21673 & actin-depolymerizing factor & AT5G59880 & & & \\
\hline Potra003719g22556 & macrophage migration inhibitory factor & AT5G01650 & & & $\mathrm{x}$ \\
\hline Potra003830g23027 & alpha type-7 & AT5G66140 & & & $\mathrm{x}$ \\
\hline Potra004406g24892 & RNA-binding protein & AT1G51510 & & $\mathrm{x}$ & \\
\hline Potra006771g25788 & NADH dehydrogenase [ubiquinone] 1 alpha subcomplex subunit & AT5G47890 & & & \\
\hline Potra008191g26111 & Glycosyl hydrolase superfamily protein & AT4G16260 & $\mathrm{x}$ & & \\
\hline
\end{tabular}

Figure 1. Populus tremula leaf physiology and morphology. A) A representative mature terminal leaf.B) A representative flushed short shoot bud with mature pre-formed leaves. C) Cross-section (15 $\mu \mathrm{m}$ thick) of a representative mature terminal leaf. D) Cross-section (10 $\mu \mathrm{m}$ thick) of a representative mature pre-formed leaf. Cross sections were embedded in 1,5\% plant agarose, sectioned using a vibratome and stained for one minute with Toluidine Blue; Magnification 200X.

Figure 2. An overview of leaf circularity, indent depth and leaf area in the Swedish Aspen (SwAsp) collection. A) Example leaves from the SwAsp collection. Each leaf is from one genotype and the set of leaves was selected to reflect the extent of variation represented among all genotypes. B) Genetic correlations among measures of leaf circularity, median indent depth and leaf area for data recorded in two common gardens (Ekebo, southern Sweden and Sävar, northern Sweden) and two years (2008 and 2011). C) Heatmap representations of leaf circularity (left), median indent depth (centre) and leaf area (right) in the two common gardens and years. Each represented value is a median calculated from 10 leaves per clonal replicate (median $\mathrm{n}=3$ ) of each genotype. In each heat map, genotypes are clustered by Euclidean distance with the population from which the genotype originates indicated by coloured bars to the left of the heat map. The values represented were used to perform the genome-wide association mapping results depicted in D. D) Intersection of the top 1,000 ranked SNPs from genome wide association mapping for the three traits measured in the two years and gardens.

Figure 3. The genomic context distribution of SNPs within the top ranked 1,000 genes identified using association mapping for leaf circularity and indent depth.

Figure 4. Selection of a set of high and low phenotype value genotypes. Genotypes with high and low phenotypic values of circularity (A ) and indent depth (B ) were selected on the basis of the top or bottom quartiles of the Best Linear Unbiased Prediction (BLUP) values for each trait. The colour intensity reflects the four quartiles of trait values with darker colours meaning higher values. The left density plots represent BLUPs for the two traits. The distribution plots in the centre depict the original trait measurements in the two gardens and the two years of sampling prior to the BLUP calculation. Gene expression assayed in young leaf buds (Mähler et al. 2017) was used to test for differential expression between genotypes within the low and high sets for each phenotypic trait. The Venn diagram shows the intersection between the significantly differentially expressed genes and the top 1,000 genes from the genome wide association study (GWAS) for the two traits.

Figure 5. Overview of gene expression in developmental series of terminal leaves. A) Principal component analysis of the 1,000 most variable genes. Data points are shaded by tissue type $(\mathrm{Pf}=$ Pre-formed; $\mathrm{T}$ $=$ terminal) and leaf number in addition to different point styles being used. Ellipses around each group indicate a 95\% confidence area for a particular sample group. B) Differential expression matrix of all sample points showing all significantly up-regulated genes $\left(P_{\text {adj }}<0.05\right)$ in the lower triangular matrix and all significantly down-regulated genes $\left(P_{\text {adj }}<0.05\right)$ in the upper triangular matrix. In the lower triangular, 
columns constitute the numerator of the differential test, while rows constitute the numerator in the upper triangular half. $\mathrm{T}=$ terminal.

Figure 6. Visualisation of differential expression within the terminal developmental series gene co-expression network. A)Transition between stage -4 and -3. B) Transition between stage -3 and -2 . C) Transition between stage -2 and -1 . D)Transition between stage -1 and 0 . E) Transition between stage 0 and 1 . F) Transition between stage 1 and 2. Nodes are sized inversely by adjusted $P$-value of a differential expression comparison at the relevant transition and shaded by the fold change (higher in red, lower in blue) with the earlier leaf number as the numerator. $P$ - values ranged from $1.519197 \mathrm{e}-104$ to $9.999962 \mathrm{e}-01$ with the highest $P$-value nodes being 100x smaller than the lowest ones.

Figure 7. Developmental expression of genes differentially expressed between phenotypic extremes. The heatmap shows the expression of the differentially expressed genes depicted in Figure 4. Expression in the terminal leaf development data set is represented for genes differentially expressed between phenotypically extreme genotype sets for circularity (A ) and indent depth (B ). Genes that were found among the top 1,000 GWAS genes are indicated by coloured lines on the left side of the heatmap. Genes are clustered hierarchical clustering using average linkage. Samples are arranged by leaf age. Values depicted are VST (VarianceStabilising Transformation) normalised expression. Clustering was performed using complete linkage and correlation-based distance.

Supplementary Figure S1. Boxplots showing genotype x environment interactions for size and shape metrics in pre-formed leaves of the SwAsp collection in common gardens at Ekebo $\left(56^{\circ} \mathrm{N}\right)$ and Sävar $\left(62^{\circ} \mathrm{N}\right)$. Each box represents a common garden in a given year with 111 to 113 genotypes in clonal replicates. (A) Leaf circularity, (B) indent depth, and (C) leaf area. GxE analysis of variance $F$ and $P$-values are provided in Table 1 Boxes annotated with the same letter are not significantly different at $P<0.05$.

Supplementary Figure S2 . Manhattan and QQ-plots for leaf area, circularity, and indent depth.

Supplementary Figure S3 . Venn diagram of the top 1,000 genes from the genome wide association analysis for the three leaf traits.

Supplementary Figure S4 . Correlation between gene expression among genotypes of the Swedish Aspen collection and leaf shape and size traits. The left panel shows the distribution for all expressed genes for each phenotypic trait. The right panel separates genes that were differentially expressed between sets of genotypes with contrasting trait values from non-differentially expressed genes.

Supplementary Figure S5. Network clusters of genes within the community expression network inferred for the terminal leaf developmental series. Clusters were defined using InfoMap. The corresponding eigengene expression profile is shown for each partition as well as representative enriched PFAM and GO categories. * indicates an adjusted $P$-value for the terms between 0.05 and $0.01,{ }^{* *}$ indicates $0.01-0.001,{ }^{* * *}$ indicates $<0.001$. Only terms in the same range of significance were included for a cluster (if any). The node size is scaled according to the node's PageRank.

Supplementary Figure S6 . Gene set enrichment analysis results for 1,000 top genes from the genome wide association (GWAS) and differentially expressed gene sets from the GWAS population (DEGs) within the terminal developmental gene co-expression network.

Supplementary Figure S7 . Heatmap of the scaled and centred gene expression values from the terminal leaf development series for all genes that were differentially expressed between genotype extreme groups for at least one of the traits, indicated by the green marks on the left.

Supplementary Figure S8 . Venn diagram of the genes differentially expressed between phenotypic extremes (high and low BLUP quartiles) of the three leaf traits.

Supplementary Figure S9. Scaled network centrality for genes that were differentially expressed between sets of genotypes with contrasting trait values and non-differentially expressed genes within the Swedish 
Aspen, terminal and pre-formed leaf development series co-expression networks. The Swedish Aspen coexpression network is from Mähler et al . (2017) and uses WGCNA-calculated network centrality scores. The leaf development network uses InfoMap scores. Both scores were scaled to uniform scale to enable comparison. Results were compared to randomised sets of genes to ensure that the observed signal was not a general pattern. Genes that were also among the top 1,000 genes identified by genome wide association mapping are indicated as coloured data points.

Supplementary Figure S10. Gene set enrichment analysis results for genome wide association and population differentially expressed gene sets within the Swedish Aspen gene co-expression network from Mähleret al. (2017).

Supplementary Figure S11. Tajima's D values for the top 1,000 genes identified by genome wide association mapping and for genes that were differentially expressed between sets of genotypes with contrasting trait values. Tajima's D values were obtained from Mähler et al.(2017). Differentially expressed genes that were also among the top 1,000 genes identified by genome wide association mapping are indicated as coloured data points.

Supplementary Table S1 . Top 1,000 genome wide associations for leaf area, circularity, and indent depth. The columns are snp: SNP name; chr: scaffold where SNP is located; pos: base-pair position of SNP on scaffold; pseudo_chr: pseudo-chromosome where SNP is located; pseudo_pos: base-pair position of SNP on pseudo-chromosome; minor_allele: minor allele of SNP; major_allele: major allele of SNP; maf: minor allele frequency of SNP; beta: effect size of the association; se: standard error of the beta; pvalue: likelihood ratio test $P$-value of the beta being different from zero; gene: gene that the SNP is located in/near (within 2 $\mathrm{kbp}$ ), if any; feature: gene feature that the SNP is located in, if any; at_gene: best BLAST hit inArabidopsis thaliana ; potri_gene: best BLAST hit inPopulus trichocarpa .

Supplementary Table S2 . Top 1,000 genes from the genome wide associations for leaf area, circularity, and indent depth. The genes were selected by rank ordering the GWAS results by association $P$-value and then picking genes from the top of the list until 1,000 unique genes had been selected.

Supplementary Table S3 . Differential expression results for the three leaf traits. In addition to the statistics from DESeq2, it has been indicated whether the gene is also an eGene in the SwAsp collection, whether the gene is part of the leading-edge subset in the gene set enrichment analysis for the trait, gene annotations, reciprocal best DIAMOND hit in A. thaliana, as well as the best DIAMOND hit in A. thaliana

Supplementary Table S4 . Node centrality statistics of all genes in the terminal leaf network. The centrality statistics computed are PageRank, closeness, betweenness, strength, eigenvector, Katz, Kleinberg's authority and Kleinberg's hub score.

Supplementary Table S5 . Gene ontology and PFAM (protein family) enrichments for all clusters with more than five members in the terminal leaf network.

Supplementary Table S6 . Normalised and raw expression values and results of all pairwise differential expression comparisons containing mean expression, $\log _{2}$ fold changes, standard error of the $\log _{2}$ fold changes, the test statistic, the test $\mathrm{P}$ value and the adjusted $\mathrm{P}$ value for all genes in all comparisons.

\section{Hosted file}

Figure1.pdf available at https://authorea.com/users/302629/articles/432738-leaf-shape-inpopulus-tremula-is-a-complex-omnigenic-trait 

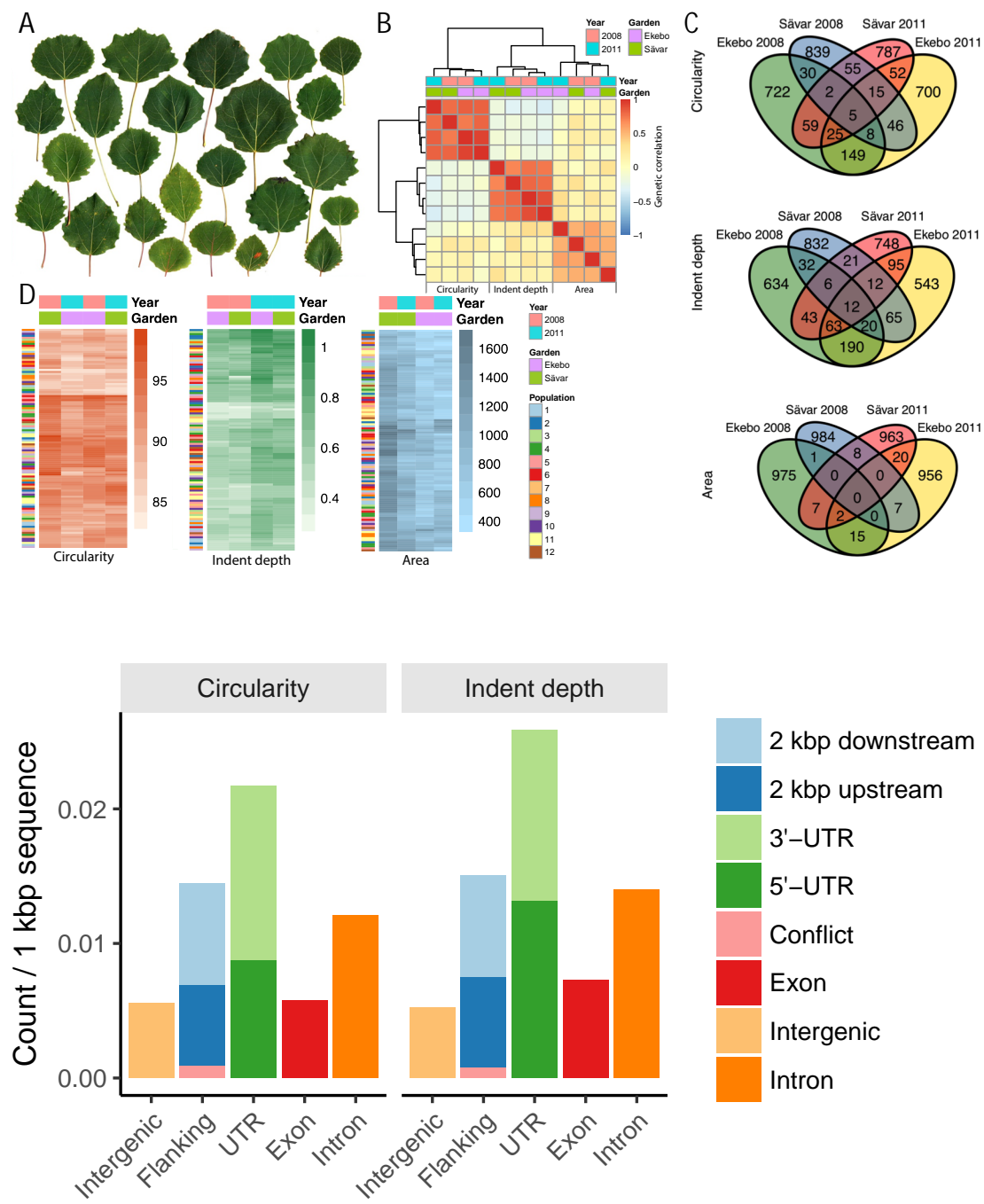

\begin{tabular}{|l|}
\hline 2 kbp downstream \\
\hline 2 kbp upstream \\
\hline 3'-UTR \\
\hline 5'-UTR \\
\hline Conflict \\
\hline Exon \\
\hline Intergenic \\
Intron
\end{tabular}



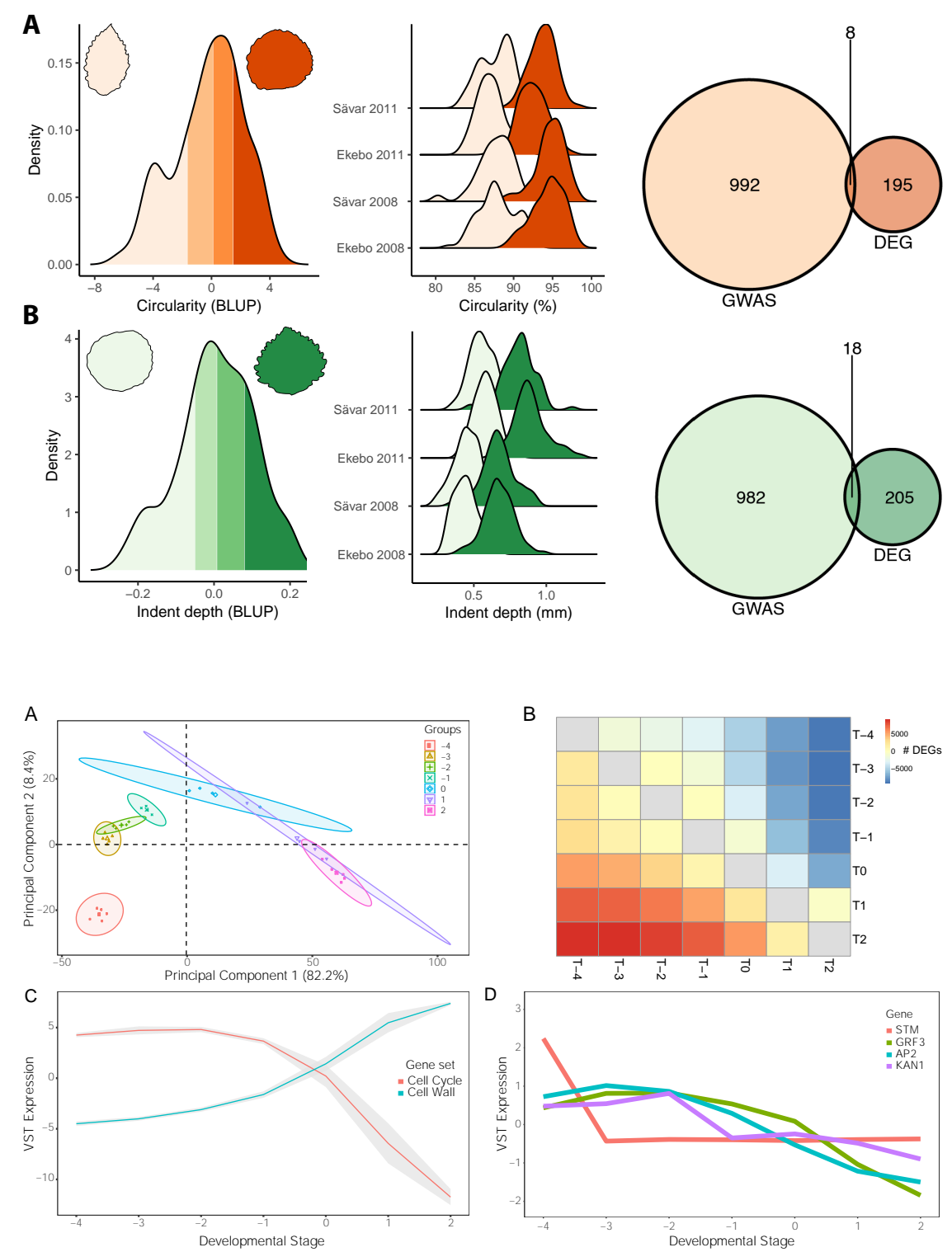

\section{Hosted file}

Figure6.pdf available at https://authorea.com/users/302629/articles/432738-leaf-shape-inpopulus-tremula-is-a-complex-omnigenic-trait 

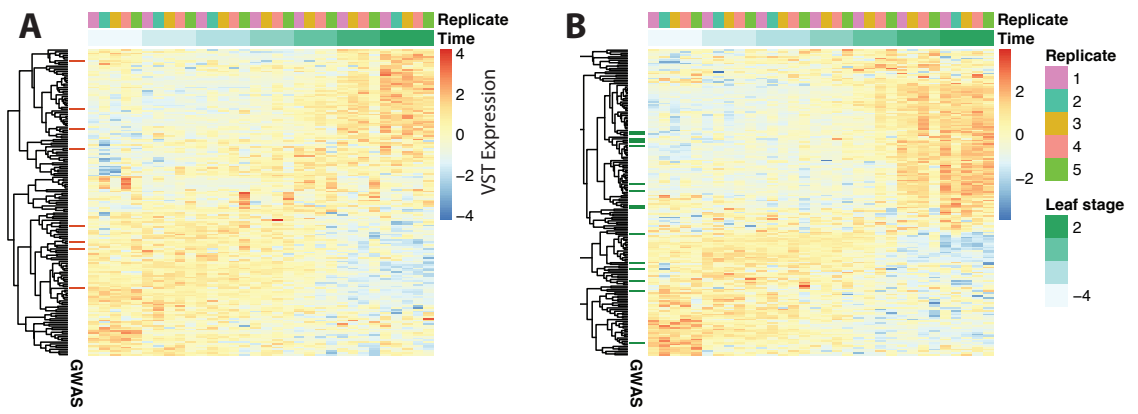